\title{
Identification and expression analysis of methyl jasmonate responsive ESTs in paclitaxel producing Taxus cuspidata suspension culture cells
}

\author{
Sangram K Lenka', Nadia Boutaoui ${ }^{1}$, Bibin Paulose ${ }^{2}$, Kham Vongpaseuth ${ }^{3,4}$, Jennifer Normanly ${ }^{2,4}$,
} Susan C Roberts ${ }^{3,4}$ and Elsbeth L Walker ${ }^{1,4^{*}}$

\begin{abstract}
Background: Taxol $^{\circledR}$ (paclitaxel) promotes microtubule assembly and stabilization and therefore is a potent chemotherapeutic agent against wide range of cancers. Methyl jasmonate (MJ) elicited Taxus cell cultures provide a sustainable option to meet the growing market demand for paclitaxel. Despite its increasing pharmaceutical importance, the molecular genetics of paclitaxel biosynthesis is not fully elucidated. This study focuses on identification of MJ responsive transcripts in cultured Taxus cells using PCR-based suppression subtractive hybridization (SSH) to identify genes involved in global pathway control.
\end{abstract}

Results: Six separate SSH cDNA libraries of paclitaxel-accumulating Taxus cuspidata P991 cell lines were constructed at three different post-elicitation time points (6h, $18 \mathrm{~h}$ and 5 day) to identify genes that are either induced or suppressed in response to MJ. Sequencing of 576 differentially screened clones from the SSH libraries resulted in 331 unigenes. Functional annotation and Gene Ontology (GO) analysis of up-regulated EST libraries showed enrichment of several known paclitaxel biosynthetic genes and novel transcripts that may be involved in MJ-signaling, taxane transport, or taxane degradation. Macroarray analysis of these identified genes unravelled global regulatory expression of these transcripts. Semi-quantitative RT-PCR analysis of a set of 12 candidate genes further confirmed the MJ-induced gene expression in a high paclitaxel accumulating Taxus cuspidata P93AF cell line.

Conclusions: This study elucidates the global temporal expression kinetics of MJ responsive genes in Taxus suspension cell culture. Functional characterization of the novel genes identified in this study will further enhance the understanding of paclitaxel biosynthesis, taxane transport and degradation.

\section{Background}

Although the supply of most plant-derived secondary metabolic products is through harvest of the whole plant, many of these commercially valuable compounds are produced in slow-growing or non-agriculturally produced species [1]. Harvest from natural sources is often insufficient to provide adequate quantities of the product of interest in a

\footnotetext{
*Correspondence: ewalker@bio.umass.edu

'Department of Biology, University of Massachusetts, Amherst, MA 01003, USA

${ }^{4}$ Plant Biology Graduate Program, University of Massachusetts, Amherst, MA 01003, USA

Full list of author information is available at the end of the article
}

sustainable manner. Plant cell culture provides an alternative production source in which environmental conditions can be more easily controlled, manipulated, and optimized to yield high quantities of these valuable products (i.e., secondary metabolites).

The anticancer drug paclitaxel (registered as a trademark Taxol ${ }^{\circledR}$ by Bristol-Myers Squibb) is a secondary metabolite with a complex chemical structure that has been approved by the Food and Drug Administration (FDA) for the treatment of breast, ovarian and lung cancers, AIDS-related Kaposi's sarcoma as well as coronary artery disease (http://www.fda.gov). The production of paclitaxel from Taxus cell cultures has been achieved by

\section{Biomed Central}


us and others [2-11]. Plant cell culture technology has received significant attention in meeting the demand for paclitaxel and this method of production of paclitaxel has been licensed by Bristol-Myers Squibb to Phyton Biotech Inc. (Fort Worth, TX).

Paclitaxel is a member of the taxane family of compounds that are produced by all species of yew. Over 400 taxanes have been reported from various Taxus species [12]. The structure of paclitaxel was determined by Wani et al., (1971) as a diterpenoid (molecular formula of $\mathrm{C}_{47} \mathrm{H}_{51} \mathrm{NO}_{14}$ ) with a highly functionalized taxane skeleton [13]. Taxanes vary in the functional groups and side-chain components attached to the tricyclic taxane skeleton. Many steps of the biosynthetic pathway have been elucidated through the isolation of genes and enzymes from Taxus tissues and cultured cells [14-16]. Using molecular approaches, the genes encoding GGPP synthase [17], 10deacetylbaccatin III-10-O-acetyl transferase [18], taxadiene synthase [19], cytochrome P450 taxane 10ß-hydroxylase [20], 3'-N-debenzoyl-2'-deoxyTaxol N-benzoyltransferase [21], baccatin III:3-amino-3-phenylpropanoyl transferase [22], taxane $13 \alpha$-hydroxylase [23], taxane 2 $\alpha$-O-benzoyltransferase [24], and taxa-4(20),11 (12)-dien-5 $\alpha$-ol-Oacetyl transferase [25] were identified.

The biotic elicitor methyl jasmonate (MJ) is an integral component of the signal transduction process that regulates the inducible defence systems of plants. Exogenous MJ is highly effective at eliciting secondary metabolite accumulation in a variety of plant cell culture systems [26]. Accordingly, paclitaxel accumulation is significantly enhanced upon MJ addition [27-31].

The non-model gymnosperm Taxus is evolutionally diverged and quite distantly related to model angiosperm species. This presents significant challenges to genomics approaches for understanding paclitaxel synthesis and regulation, and necessitates the generation of Taxus-specific genetic information in order to establish gene-to-metabolite links. Genetic information for Taxus species has begun to emerge from transcriptome sequencing of Taxus organs (needles, bark, cambial meristematic cells etc.) [3234]. However, a differentially-regulated transcripome of paclitaxel accumulating cultured cells in controlled environmental conditions, and in response to secondary metabolite elicitors like MJ, needs to be characterized. Here we describe the results from transcript profiling experiments in Taxus culture cells to identify genes involved in global pathway control, including not only potential paclitaxel biosynthetic genes, but also genes that may be involved in MJ-signaling, taxane transport, or taxane degradation. Using a PCR-based suppression subtractive hybridization strategy, MJ responsive ESTs were isolated from the paclitaxel-accumulating P991 Taxus cuspidata cell line. Expression analysis of randomly selected differentiallyregulated ESTs from the MJ-treated $\mathrm{SSH}$ libraries was also performed using macroarrays. Computational functional annotation and Gene Ontology (GO) analysis of up-regulated EST libraries show enrichment of several known paclitaxel biosynthetic genes as well as novel transcripts. RT-PCR analysis of a set of 12 candidate genes further confirmed the MJ-induced gene expression in the paclitaxel accumulating cell line. Hence, this study is useful in expanding our understanding of the molecular genetics of the complex paclitaxel biosynthesis process.

\section{Results}

\section{Differential screening of MJ-responsive ESTs}

P991 is a well characterized T. cuspidata cell line that produces higher amounts of taxanes after MJ elicitation $[27,35]$. This cell line was used to detect differentially expressed MJ responsive transcripts with a PCR-based cDNA subtraction method. Six distinct driver/tester combinations were used to construct six different $\mathrm{SSH}$ libraries. To generate libraries enriched for up-regulated genes, the combinations of driver/tester were as follows:

1) Driver = unelicited control at 6 hour time point; tester $=$ MJ-elicited at 6 hour time point

2) Driver $=$ unelicited control at 18 hour time point; tester $=$ MJ-elicited at 18 hour time point

3) Driver $=$ unelicited control at 5 day time point; tester $=$ MJ-elicited at 5 day time point

In contrast, to produce SSH libraries enriched for downregulated genes the opposite driver/tester combination (i.e. MJ-elicited driver and unelicited tester) was used with the same cDNA samples. Single pass sequencing was carried out on 288 recombinant clones each from the MJ up- and down-regulated SSH libraries (96 clones each from $6 \mathrm{~h}, 18 \mathrm{~h}$ and 5 day time point of both the up/down regulated libraries). Among the 576 clones sequenced, 548 were considered for contig assembly and annotation after excluding vector sequence contamination. All the EST sequences from the three up-regulated libraries were used together for contig assembly with CAP3. Similarly, the ESTs from the three down-regulated libraries were used together for asssembly of down-regulated contigs. CAP3 assembly of the ESTs

\section{Table 1 Overview T. cuspidata P991 SSH libraries}

\begin{tabular}{lll}
\hline Features & $\begin{array}{l}\text { Up-regulated } \\
\text { libraries }\end{array}$ & $\begin{array}{l}\text { Down Regulated } \\
\text { libraries }\end{array}$ \\
\hline Number of clones sequenced & 288 & 288 \\
Number of clean ESTs & 283 & 265 \\
Number of non-redundant ESTs & 155 & 176 \\
Total number of contigs & 32 & 23 \\
Total number of singletons & 123 & 153 \\
Number of randomly chosen & 128 & 128 \\
ESTs used for macroarray analysis & & \\
\hline
\end{tabular}


generated $32 \mathrm{MJ}$ up-regulated contigs, 123 singletons and 23 MJ down-regulated contigs, 153 singletons (Table 1) [36]. Hence, the up-regulated libraries contain 155 unigenes (combination of contigs, Table 2, Additional file 1: Table S1 and singletons, Supplemental 2) and 176 unigenes are represented in the down-regulated libraries (Additional file 2: Table S2). The mean length of the ESTs obtained from all the libraries was $658 \mathrm{bp}$, which is adequate for sequence similarity based functional classification.

\section{Functional classification of the unigenes}

Putative functional annotation of the unigenes (Additional file 2: Table S2) was assigned by BLASTX analysis against the GenBank non-redundant database for sequence similarity (E-value $\leq 10^{-2}$ ). Unigenes showing BLASTX homology at $\mathrm{E}>10^{-2}$ were designated as unigenes with no match in the database. Approximately $47 \%$ of the unigenes fell into this category, and may be specific to Taxus species. Approximately $12 \%$ of the sequences represented evolutionarily conserved proteins whose function is not known. Many of the known paclitaxel biosynthetic genes were induced, as expected. Several novel transcripts that are similar, but not identical, to known taxane biosynthetic enzymes were identified. These included two genes that are similar to known taxane acyl transferases and five genes that are related to known taxane hydroxylase. Experimental characterization of these novel genes would be needed to definitively determine the exact acylation or

Table 2 Identity and description of contigs obtained from T. cuspidata up-regulated SSH libraries

\begin{tabular}{|c|c|c|c|c|c|}
\hline Contig & Accession No & Match with Accession No & Description & Query coverage (\%) & E Value \\
\hline 03 & HE799317 & ABR16435 & Predicted Cytochrome P450 & 66 & $5.00 \mathrm{E}-72$ \\
\hline 08 & HE799322 & BAF46017 & Putative class I chitinase & 35 & $5.00 \mathrm{E}-64$ \\
\hline 21 & HE799335 & AAR13860 & Taxadiene synthase & 92 & 4.00E-61 \\
\hline 25 & HE799339 & ABK26019 & Unknown & 34 & $1.00 \mathrm{E}-42$ \\
\hline 30 & HE799344 & BAD90813 & Thaumatin-like protein & 76 & $2.00 \mathrm{E}-37$ \\
\hline 04 & HE799318 & AAZ41362 & Taxadiene synthase & 94 & 5.00E-30 \\
\hline 28 & HE799342 & XP_002264098 & hypothetical protein & 49 & $2.00 \mathrm{E}-27$ \\
\hline 26 & HE799340 & CAI56321 & putative leucoanthocyanidin reductase & 68 & $3.00 \mathrm{E}-25$ \\
\hline 18 & HE799332 & XP_002516771 & putative Lipoxygenase & 59 & $5.00 \mathrm{E}-25$ \\
\hline 15 & HE799329 & AAS89065 & putative taxoid hydroxylase & 65 & $8.00 \mathrm{E}-22$ \\
\hline 17 & HE799331 & ABK21418 & Unknown & 72 & $2.00 \mathrm{E}-23$ \\
\hline 29 & HE799343 & ACH59388 & putative Chalcone synthase & 36 & $3.00 \mathrm{E}-21$ \\
\hline 12 & HE799326 & AAO85809 & putative S-adenosylmethionine synthetase & 43 & $3.00 \mathrm{E}-20$ \\
\hline 09 & HE799323 & BAD10865 & $\begin{array}{l}\text { predicted 1-aminocyclopropane-1-carboxylic } \\
\text { acid oxidase }\end{array}$ & 79 & 7.00E-18 \\
\hline 11 & HE799325 & ZP_03319418 & Hypothetical protein & 55 & $5.00 \mathrm{E}-16$ \\
\hline 01 & HE799315 & AAT73199 & predicted taxoid acyltransferase & 81 & $9.00 \mathrm{E}-16$ \\
\hline 14 & HE799328 & XP_002264858 & Hypothetical protein & 66 & $9.00 \mathrm{E}-16$ \\
\hline 07 & HE799321 & XP_002973748 & Hypothetical protein (XP_002973748) & 32 & $1.00 \mathrm{E}-15$ \\
\hline 16 & HE799330 & XP_001768181 & similar to predicted protein (XP_001768181) & 47 & $2.00 \mathrm{E}-15$ \\
\hline 27 & HE799341 & ABR16535 & Unknown & 16 & $7.00 \mathrm{E}-15$ \\
\hline 02 & HE799316 & ABR17205 & putative DNA binding protein & 79 & $2.00 \mathrm{E}-13$ \\
\hline 05 & HE799319 & ACN40018 & Unknown & 60 & 2.00E-09 \\
\hline 32 & HE799346 & ADQ12769 & Cytochrome P450 & 41 & $3.00 \mathrm{E}-09$ \\
\hline 06 & HE799320 & ABK22210 & Unknown & 39 & $8.00 \mathrm{E}-07$ \\
\hline 23 & HE799337 & ADK74829 & putative Immunomodulatory protein & 13 & $3.00 \mathrm{E}-05$ \\
\hline 20 & HE799334 & ABK23181 & Unknown & 11 & $3.00 \mathrm{E}-04$ \\
\hline 10 & HE799324 & ABK24846 & putative Gibberellin 3-beta-hydroxylase & 38 & 0.001 \\
\hline 31 & HE799345 & ADM79415 & similar to Dormancy/auxin associated-like protein & 27 & 0.001 \\
\hline 22 & HE799336 & YP_260089 & Cytotoxin Mcf & 41 & 0.003 \\
\hline 19 & HE799333 & XP_002283086 & hypothetical protein & 8 & 0.005 \\
\hline 13 & HE799327 & - & No hits in the database & - & - \\
\hline 24 & HE799338 & - & No hits in the database & - & - \\
\hline
\end{tabular}


hydroxylation reactions catalyzed by the encoded enzymes.

Functional classification of $\mathrm{MJ}$ up-regulated unigenes was determined based on GO analysis for biological process, molecular function and cellular component (Additional file 3: Figure S1a-c). Among the biological process category, unigenes belonging to oxidation-reduction processes (GO:0055114), responses to stimulus (GO:0050896), small molecule biosynthetic processes (GO:0044283) and paclitaxel biosynthetic processes (GO:0042617) were over-represented. In the molecular function category, oxidoreductase activity (GO:0016491) was most significantly enriched, followed by iron ion binding (GO:0005506) and transferase activity (GO:0016740). Finally, in the cellular component category, proteins associated with intracellular organelles (GO:0043229), membranes (GO:0016020) and cytoplasmic parts (GO:0044444) were most enriched. KEGG pathway analysis of the encoded proteins further indicated MJ-induced paclitaxel, L-methionine and oxylipin biosynthesis pathways in the up-regulated SSH libraries. There were too few truly down-regulated unigenes available (see below) for GO analysis of down regulated transcripts.

\section{Evaluation of MJ-responsive differential expression using macroarrays}

Probe sets designed from up- and down-regulated SSH libraries were spotted manually as macroarrays on nylon membranes and hybridized with different RNA targets. Of the 256 randomly chosen ESTs in the macroarray, 81 (71 up- and 10 down- regulated) ESTs showed a significant change in intensity following MJ elicitation (Additional file 4: Table S3). One set of genes showed the highest up-regulation trend at early time points (6h and 18h) following MJ elicitation (Figures 1a, b and 2 ), while MJ responsive expression of a separate set of genes exhibited a difference in expression pattern at the late time point (5 day; Figures 1c, 2). On the other hand, the majority (92.2\%) of MJ down-regulated unigenes were found to be false positive SSH clones (Additional file 4: Table S3, Additional file 5: Figure S2, Additional file 6: Figure S3, Additional file 7: Figure S4) based on this analysis. Hierarchical clustering analysis of the temporal differential gene expression patterns of the individual ESTs in the macroarray (Figure 2) clearly demonstrated the distinctions between the MJ-responsive early, middle and late gene regulation.

\section{Confirmation of MJ-induced expression by RT-PCR}

Twelve randomly selected genes from the up-regulated SSH library were evaluated for transcriptional response in another paclitaxel-accumulating line T. cuspidata P93AF using semi-quantitative RT-PCR. All 12 genes were upregulated at $6 \mathrm{~h}$ after $\mathrm{MJ}$ elicitation, further supporting the early overall response observed in the macroarray (Figure 3). Transcript levels for flavonol synthase (D5_88H) and the pyridine nucleotide-disulfide oxidoreductase family protein (18R_32H) went down after 6h. Cytochrome P450 (contig 32), unknown (contig 6), ACC oxidase (contig 9), leucoanthocyanidin reductase (contig 26) and lipoxygenase (contig 18) were elevated at both $6 \mathrm{~h}$ and $18 \mathrm{~h}$. However, semi-quantitative RT-PCR could not detect transcripts of these genes on day 5 after elicitation. Transcripts encoding cytochrome P450 (contig3), quinone oxidoreductase-like protein (18R_44D), hypothetical protein (contig 16), dirigent-like protein (18R_17A) and short-chain dehydrogenase/reductase family protein were at elevated levels on day 5 as well as at early time points. Elevated expression of these randomly chosen sequences in the $T$. cuspidata P93AF cell line upon MJ-elicitation further supported that regulated genes were successfully identified using the subtracted cDNA libraries from cell line P991.

\section{Discussion}

MJ potentiates secondary metabolite accumulation in a variety of plant suspension cell culture systems, and is now a widely-used augmenting tool for bio-active molecule production [26]. Accordingly, paclitaxel accumulation is significantly enhanced with MJ elicitation in $T$. cuspidata suspension cultured cells [27,37-40]. MJ is also implicated in specific induction of a wide range of secondary metabolite genes involved in biotic-stress response and wounding [41]. Conversely, primary metabolic genes such as those involved in photosynthesis, electron transport and cytoskeletal organization are either down-regulated or unaffected by MJ elicitation [42]. MJ elicitation and targeted cloning approaches have been effectively used in Taxus to identify specific taxane biosynthetic genes $[43,44]$. Therefore, an up-regulated cDNA library of MJ treated cells will presumably contain genes related to secondary metabolism, including paclitaxel biosynthesis.

SSH is a powerful genomics technique that enriches differentially regulated genes and has mostly been used to identify transcripts expressed in contrasting environmental conditions. SSH is a suitable approach in Taxus where the genomic information is currently scarce. We used the PCR-based SSH approach to identify MJ responsive (up- and down-regulated) transcripts that precede paclitaxel accumulation in the paclitaxel accumulating $T$. cuspidata P991 cell line. Putative up-regulation of as many as 155 unigenes and down-regulation of 176 unigenes of diverse functional groups suggested involvement of a spectrum of genes regulating paclitaxel biosynthesis, MJ-signaling and response, and potentially, taxane transport and taxane degradation. The up-regulated $\mathrm{SSH}$ library contained several previously known positively regulated genes that are likely to be directly involved in paclitaxel synthesis (Additional file 2: Table S2). For 


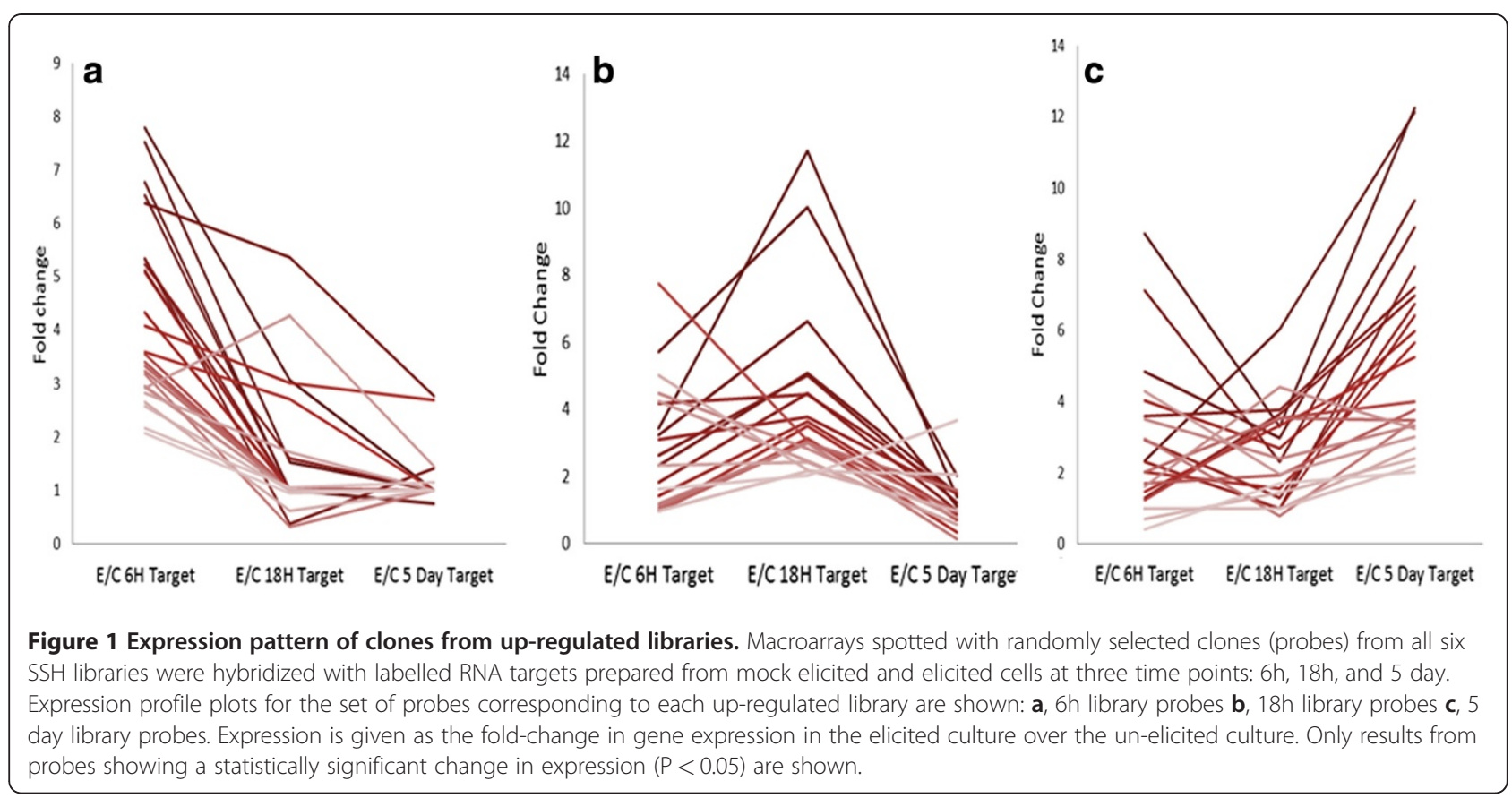

instance, putative taxadiene synthase, taxane hydroxylase and taxane acyl transferases having strong sequence similarity with the corresponding known paclitaxel biosynthetic pathway genes were detected in the up-regulated library. Taxadiene synthase catalyses the first committed step of paclitaxel biosynthesis in Taxus by cyclization of the linear isoprenoid substrate geranyl geranyl diphosphate (GGPP) to form taxa-4 (5),11(12)diene [19]. Four unigenes representing different isoforms of putative taxadiene synthase were upregulated in the library. It will be interesting to unravel the individual contribution of each of these taxadiene synthase genes towards paclitaxel production. Another paclitaxel biosynthetic gene, taxoid 2- $\alpha$-hydroxylase, produces $2 \alpha$-hydroxy taxoids as starting materials for subsequent acylation at the $\mathrm{C}_{2}$-position of the taxane core that ultimately bears a benzoyl group, an important paclitaxel pharmacophore [45]. Five novel putative taxoid hydroxylases that are similar but not identical to known taxoid hydrolases (taxoid 2-alpha hydroxylase, taxoid 5-alpha hydroxylase, and taxoid 7-beta hydroxylase) were detected in the up-regulated library. Each might function to hydroxylate taxoids at specific positions. Similarly, novel taxane acyl transferases are not only important targets for genetic manipulation to improve paclitaxel production in genetically engineered host systems but also provides a means of attaching modified aroyl groups to taxoid precursors to improve drug efficacy $[18,21,25,46]$. Characterization of the two newly identified candidate acyl transferases in the library will further suggest roles for these novel enzymes in paclitaxel biosynthesis.

Apart from the paclitaxel biosynthesis genes, up-regulated genes that are potential candidates for regulation of the MJ response were also obtained. One such gene encodes ACC oxidase (1-aminocyclopropane-1-carboxylate oxidase), which is the final step in the synthesis of the plant hormone ethylene [47]. Ethylene is known to be produced following MJ elicitation, and to function in suppression of the MJ signal. An increased production of paclitaxel upon application of ethylene synthesis inhibitors to elicited Taxus cultures was reported by Zhang et al., (2003) [48]. Disruption of this ACC oxidase gene may allow the MJ signal to be strengthened, which may lead to increased paclitaxel accumulation.

The library was also enriched with up-regulated genes that presumably have no direct role in paclitaxel accumulation. Good examples are the putative class I chitinase, thaumatin (also known as PR-5); chalcone synthase, and defensin, all of which are defense-related genes that have well-characterized roles in model plant species $[49,50]$. These genes are known to be induced by MJ in other plant species and thus are unlikely to be related to the synthesis of taxanes, which are unique to Taxus species. For several transcripts, a reliable prediction of general biochemical activity can be made, but predictions neither suggest nor challenge a role in paclitaxel synthesis. Genes in this class include three cytochrome P450 genes that lack significant similarity to the several known taxane biosynthetic pathway P450s, two distinct lipoxygenase genes, and a few other 


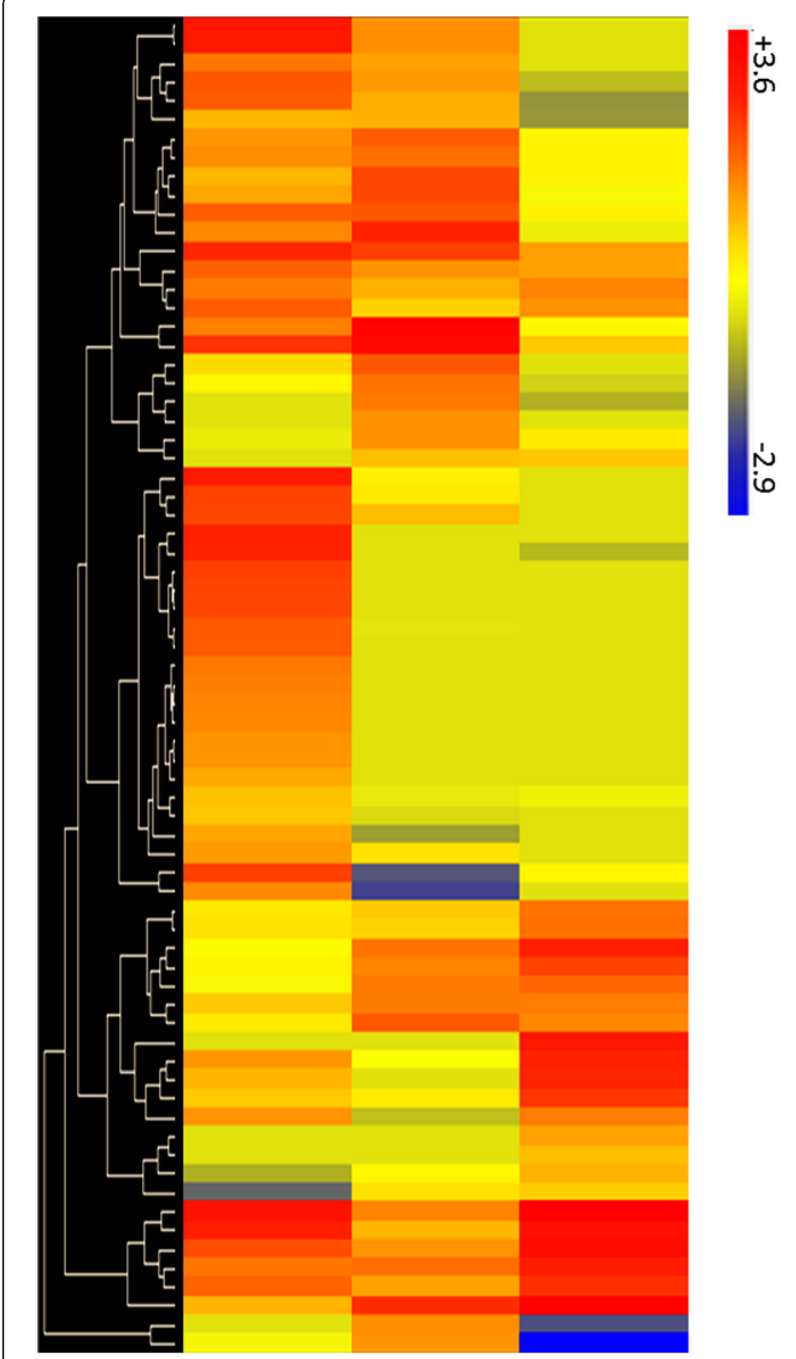

Figure 2 Heat map showing overall expression patterns of differentially regulated genes from up-regulated libraries. Hierarchical clustering of significantly MJ up-regulated genes was performed using average linkage and Euclidean distance as a measurement of similarity. Only results from probes showing a statistically significant change in expression for at least one time point $(P<0.05)$ are shown.

enzyme encoding genes. Any of these genes could participate in the uncharacterized steps of the paclitaxel biosynthetic pathway (e.g., hydroxylation reactions and oxetane ring formation), but could equally well be unrelated to paclitaxel synthesis. Further characterization will be necessary to determine whether these genes have direct relevance to paclitaxel biosynthesis.

A large class of genes (59\%) have functions that cannot be predicted based on sequence similarity information. These genes may be involved in important but uncharacterized processes of MJ-signaling, taxane transport or taxane degradation. As many as $47 \%$ of these ESTs showing no sequence homology with any of the available Genbank sequences may be specific to Taxus species. The other $12 \%$ of sequences are similar to genes described as encoding hypothetical proteins or proteins with unknown function. Enrichment of unigenes belonging to various biological process categories (GO), such as oxidation-reduction process (GO:0055114), response to stimulus (GO:0050896), small molecule biosynthetic process (GO:0044283) and paclitaxel biosynthetic process (GO:0042617), provide strong molecular evidence that the SSH-based MJ elicited transcript screening is a good method to identify genes related to paclitaxel metabolism. Nevertheless, this strategy did not enable the isolation of all the known paclitaxel metabolism genes indicating that the collection of MJ-inducible genes identified here is not comprehensive. Sequencing of the total MJ-induced transcriptome will be required to identify transcripts for every gene related to paclitaxel accumulation.

In addition to confirming up-and down-regulated genes, macroarrays identified false positive clones that appeared in the libraries but were not actually differentially regulated in response to MJ. Although most genes identified as being up-regulated were confirmed as such by macroarray analysis, the false positive rate in the downregulated libraries was $92.2 \%$. SSH libraries of highly complex eukaryotes have often been reported to contain high percentages (30-50\%) of false positive clones that escaped subtraction $[51,52]$. These high false positive rates are usually attributed to non-specific annealing of primers, partial hybridization of driver that allows tester amplification and non-specific annealing of suppression adaptors [53]. While any of these could have been factors in producing the high false positive rate in our down-regulated libraries, we also note the possibility that there could simply be very few genes that are down-regulated by MJ in the Taxus cultures. If few or no down-regulated transcripts occur, then the SSH approach is certain to be unsuccessful, and will identify a random set of highly expressed (but not differentially regulated) genes. Genes encoding cell wall proteins, ribosomal proteins and other highly expressed genes are well represented in the down-regulated libraries. The high false positive rate in the down-regulated libraries here indicates that the genes observed were not actually downregulated by $\mathrm{MJ}$.

The gene expression kinetics revealed differential temporal regulation of $\mathrm{MJ}$ responsive genes. Up-regulation of many of the MJ induced genes at early time points (6h) was highest, followed by middle time point (18h). MJ-responsive up-regulation of most genes, however, did not persist into the latest time point 5 day (Figure 2). However, a subset of late-induced genes was observed. In agreement to this study, similar expression trend was also observed previously in MJ-induced temporal expression kinetics of taxol biosynthesis genes in P991 cultured cells [27]. This trend was also observed in the 


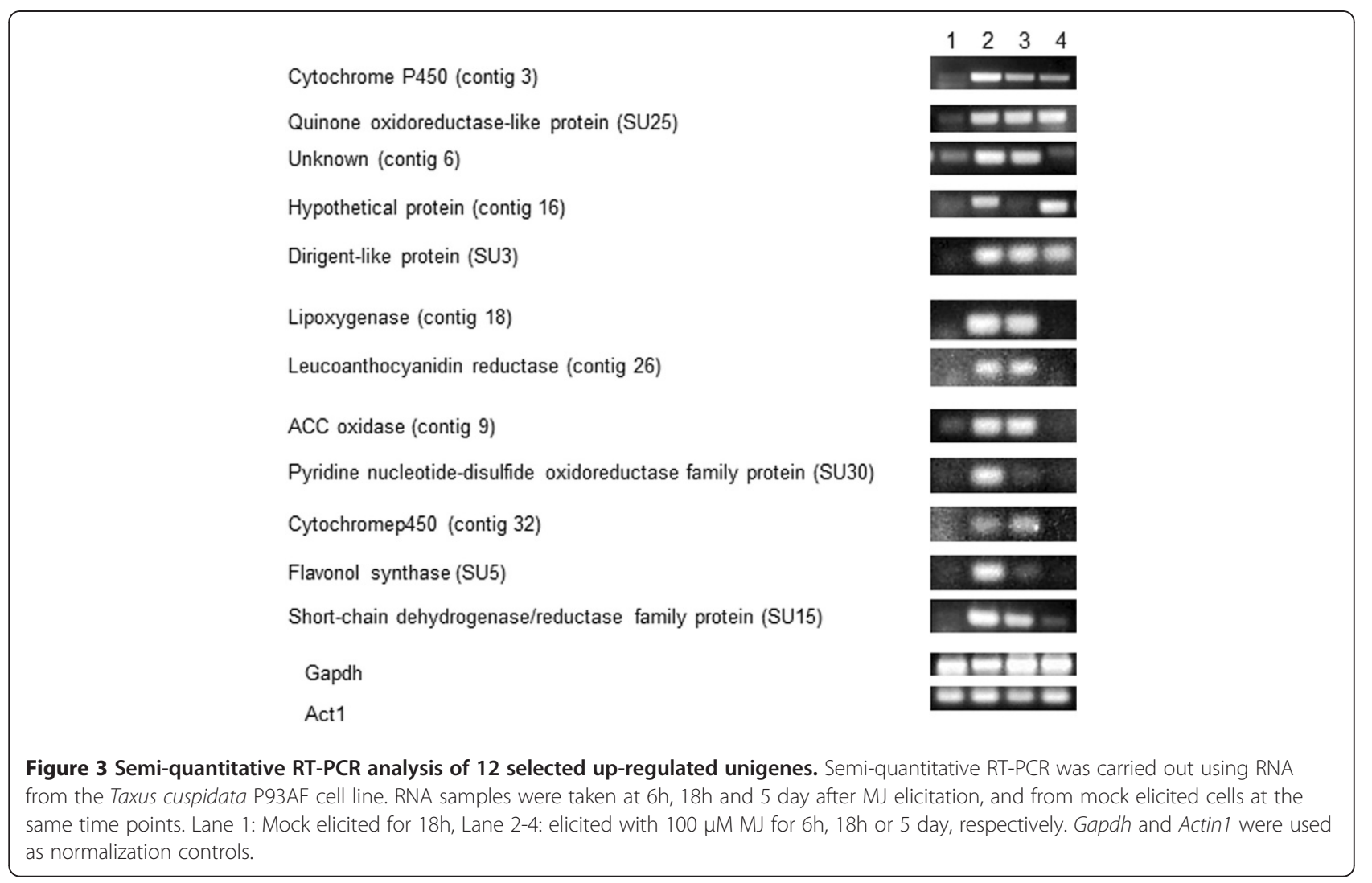

paclitaxel-producing T. cuspidata P93AF cell line, as semi-quantitative RT-PCR showed that all 12 genes were up-regulated at $6 \mathrm{~h}$ but transcripts of only 5 of them were detected on day 5 . These 12 genes tested here by RT-PCR do not represent any known genes related to taxol biosynthesis, but rather were selected as novel transcripts identified in this study. Furthermore, use of a second paclitaxel-producing cell line (P93AF) demonstrates the reproducibility of the gene expression changes occurring in Taxus cultured cells. Paclitaxel and other taxanes accumulate in the cultured cells at time points after 24h [27]. Therefore, persistent translated gene products of early $\mathrm{MJ}$ induced genes appear to play a pivotal role in taxane biosynthesis, while late MJ induced genes may be facilitating other functions such as taxane transport and degradation. Genes involved either in intracellular or extracellular paclitaxel transport and degradation in Taxus have not yet been characterized [54], but may be present among the ESTs identified here that could not be assigned functions based on molecular similarity to known proteins.

\section{Conclusions}

Several genes were differentially regulated in response to MJ in Taxus cuspidata cell lines. Among these genes, paclitaxel biosynthetic pathway genes were specifically induced in response to MJ. A majority of the up-regulated genes followed specific temporal expression kinetics. SSH was not sensitive enough to isolate tightly regulated down regulated genes. Functional characterization of the novel genes identified in this study will further enhance the understanding of paclitaxel metabolism and its regulation.

\section{Methods}

\section{Cell culture}

Taxus cuspidata cell line P991 was used for SSH library preparation and macroarray screening and cell line Taxus cuspidata P93AF was used for RT-PCR analysis. All cells were obtained as a gift from Dr. D. Gibson at the U.S. Plant Soil and Nutrition laboratory, USDA (Ithaca, NY). Cell cultures were maintained as described in Nims et al., (2006) [27]. MJ (100 $\mu \mathrm{M}$ final concentration) dissolved in $50 \% \mathrm{EtOH}$ was added to suspension cultures on day 7 after subculture. Control (unelicited) cells were subjected to mock elicitation by adding an equal volume of $50 \% \mathrm{EtOH}$. Cells were harvested from elicited and unelicited cultures at 6 hours, 18 hours and 5 day postelicitation.

\section{RNA purification}

Cells were collected by filtration from the media through Miracloth $^{\circledR}$ (EMD Biosciences, San Diego, CA), flash frozen 
in liquid nitrogen and stored at $-80^{\circ} \mathrm{C}$ in polypropylene tubes. Total RNA was extracted using guanidinium isothyocyanate and separated through a cesium chloride $(\mathrm{CsCl})$ phase [55]. Poly(A) RNA was extracted using Poly(A) Purist MAG Purification kit (Ambion, Austin, TX). Quality checks of poly (A) RNA preparations were made by PCR using primers specific for $18 \mathrm{~S}$ rRNA [56]. These primers were: PtMGB-18S-f (AGCCTTGCGCTGGCG), Pt-MGB-18S-r (TGCCCTATCAACTTTCGATGGT). Quantification of total RNA and poly(A) RNA was performed with the Ribogreen fluorometric assay (Molecular Probes, Eugene, OR).

\section{Suppression subtractive hybridization (SSH) libraries}

Suppression Subtractive Hybridization (SSH) libraries using control and MJ-elicited samples from Taxus cuspidata cell line P991 at 6 hours, 18 hours and 5 day postelicitation were generated using the Clontech PCR-Select cDNA Subtraction Kit (Takara Bio Inc., Clontech, Mountain View, CA) according to the manufacturer's instructions. For each SSH library two different double stranded cDNA pools (a 'tester' and 'driver') were used. Mirror orientation selection (MOS) was then performed to reduce background molecules [53]. Library products were separated on agarose gels. For each library, products below and above 500 base pairs were purified separately using Gel Purification Kit (Qiagen, Valencia, CA) and cloned into TOPO 2-1 vector (Invitrogen, Carlsbad, CA).

\section{Sequence analysis of the cloned ESTs}

Plasmids from independent clones were isolated and single pass sequencing was carried out. All EST sequences were screened through VecScreen software (NCBI:http:// www.ncbi.nlm.nih.gov) to remove vector sequence contamination. Once cleaned of vector sequence, all the EST sequences from each up-regulated library and from each down regulated library were used separately for contig assembly with CAP3 [36]. The ESTs were grouped into contigs and singletons and were termed as unigenes (Accession No HE799315-HE799645, Additional file 8: Table S4). The unigene sequences were used to search for homology using the default setting of BLASTX program at NCBI. Each unigene was classified as a protein with known function, a protein with unknown function or a protein with no sequence match in the database. Furthermore, sequences with BLASTx hits were annotated according to gene ontology (GO) terms using Blast2GO software (www.blast2go.org;[57]). The TrEMBL section of UniProtKB (http://www.uniprot.org) database was used to perform, enzyme class and pathway analysis.

\section{Expression analysis by macroarray}

Macroarrays constructed for this study comprised 256 $\mathrm{SSH}$ fragments from both the up-regulated and downregulated libraries (128 up and 128 down). Bacterial cultures were grown overnight in LB media containing $50 \mu \mathrm{g} / \mathrm{mL}$ kanamycin. Plasmid DNA was purified using Qiaprep-miniprep (Qiagen, Valencia, CA). SSH fragments were amplified by PCR using adapter specific primers. Amplification of fragments via PCR was performed using ExTaq Hot Start Polymerase System (Takara Mirus Bio, Madison, WI). PCR conditions were as follows: $95^{\circ} \mathrm{C}$ for 2 minutes followed by $25 \mathrm{X}$ : 30 seconds, $95^{\circ} \mathrm{C}$; $30 \mathrm{sec}-$ onds, $62^{\circ} \mathrm{C} ; 2$ minutes, $72^{\circ} \mathrm{C}$. This was then followed by $1 \mathrm{X}$ : 5 minutes, $72^{\circ} \mathrm{C}$. PCR products were precipitated, dissolved in water and denatured in $0.01 \%$ sodium dodecyl sulfate (SDS), $0.2 \mathrm{~N} \mathrm{NaOH}$ at $72^{\circ} \mathrm{C}$ for 15 minutes. Denatured PCR products were printed in duplicate on Zetaprobe membranes (BioRad, Hercules, CA) using hand held 96-pin replicating tool (V\&P Scientific, San Diego, CA). Macroarray blots were submerged in neutralization solution $(0.5 \mathrm{M}$ Tris $\mathrm{pH} 7.4,1.5 \mathrm{M} \mathrm{NaCl})$, then in $6 \mathrm{X}$ sodium citrate/sodium phosphate (SCP) for 1 minute prior to UV cross-linking. Pre-hybridization of the membranes occurred for eight hours in 10\% dextran sulfate (average molecular weight of $500,000 \mathrm{kD}$; G.E. Health, Piscataway, $\mathrm{NJ})$, 5.5X SCP, $0.9 \% n$-lauroyl sulfate-sodium salt and 0.5 $\mathrm{mg} / \mathrm{ml}$ heparin. Targets were labelled with ${ }^{32} \mathrm{P}$ dCTP (G.E. Healthcare Bio-Sciences, Piscataway, NJ) using Superscript $^{\mathrm{TM}}$ II Reverse Transcriptase (RT) (Invitrogen, Carlsbad, CA) from 300 ng polyA RNA. Hybridization was performed at $65^{\circ} \mathrm{C}$ for $18 \mathrm{H}$, followed by washing twice with $2 \mathrm{X}$ SCP, $0.1 \%$ SDS for 15 minutes then twice using $0.2 \mathrm{X}$ SCP and $0.2 \%$ SDS for 15 minutes at $65^{\circ} \mathrm{C}$. Blots were exposed to phosphorimager screen for $24-48 \mathrm{H}$ then read by FLA-5000 phosphorimager (Fujifilm Medical Systems Inc., Stamford, CT). Probes were stripped off of blots up to three times using $0.1 \%$ SDS at $100^{\circ} \mathrm{C}$. Blots were checked for residual hybridization by exposing to film for $24 \mathrm{H}$ before re-hybridizing with a different target cDNA.

Signal intensities of each spot were captured using VisualGrid (GPC Biotech Inc, Waltham, MA). Following local background subtraction, the intensity values for each spot were globally normalized using LOWESS (locally weighted polynomial regression [58]) within and across all four technical replicate experiments. The relative fold change expression signal obtained from each clone found significant in one tailed student t-test in response to $\mathrm{MJ}$ over mock elicitation were considered for further analysis ( $\geq 2$ fold for up and down). Additionally, relative fold change of three or more independent ESTs corresponding to a particular contig were analysed using Q-test with $95 \%$ confidence for finding outlying data points.

\section{Gene expression analysis by RT-PCR}

Total RNA was isolated from Taxus cuspidata P93AF cell line at $6 \mathrm{~h}, 18 \mathrm{~h}$ and 5 day after MJ elicitation as well 
as from unelicited cells (18 h) using RNeasy Plant Mini kit (Qiagen). First strand cDNA was synthesized using Affinityscript $^{\mathrm{TM}}$ cDNA synthesis kit (Stratagene) from $3 \mu g$ total RNA following the manufacture's protocol. Primers (Additional file 9: Table S5) for each subtracted cDNA were designed using Primer3 (http://fokker.wi. mit.edu/primer3/input.htm). A touchdown PCR was performed for all the transcripts with annealing temperature varying from $64^{\circ} \mathrm{C}$ to $52^{\circ} \mathrm{C}$. The number of cycles used to amplify each fragment was variable in order to detect the corresponding amplicon during linear amplification.

\section{Additional files}

Additional file 1: Table S1. The duplicate sequences obtained from the three up-regulated libraries corresponding to each contig.

Additional file 2: Table S2. Identity and description of unigenes derived from up- and down- regulated T. cuspidata SSH libraries.

Additional file 3: Figure S1. Gene Ontology mapping. GO mapping for Taxus cuspidata up-regulated unigenes by (a) biological process, (b) molecular function, and (c) cellular components.

Additional file 4: Table S3. Macroarray data from both the up- and down-regulated libraries hybridised with various RNA targets.

Additional file 5: Figure S2. Pattern of expression of clones from down-regulated libraries. Macroarrays spotted with randomly selected clones (probes) from all six SSH libraries were hybridized with labelled RNA targets prepared from mock elicited and elicited cells at three time points: $6 \mathrm{~h}, 18 \mathrm{~h}$, and 5 day. Expression profile plots for the set of probes corresponding to each down-regulated library are shown: $6 \mathrm{~h}$ library probes. Expression is given as the fold-change in gene expression in the elicited culture over the un-elicited culture. Only results from probes showing a statistically significant change in expression $(P<0.05)$ are shown.

Additional file 6: Figure S3. Pattern of expression of clones from down-regulated libraries. Macroarrays spotted with randomly selected clones (probes) from all six SSH libraries were hybridized with labelled RNA targets prepared from mock elicited and elicited cells at three time points: $6 \mathrm{~h}, 18 \mathrm{~h}$, and 5 day. Expression profile plots for the set of probes corresponding to each down-regulated library are shown: $18 \mathrm{~h}$ library probes $\mathbf{C}$. Expression is given as the fold-change in gene expression in the elicited culture over the un-elicited culture. Only results from probes showing a statistically significant change in expression $(P<0.05)$ are shown.

Additional file 7. Figure S4. Pattern of expression of clones from down-regulated libraries. Macroarrays spotted with randomly selected clones (probes) from all six SSH libraries were hybridized with labelled RNA targets prepared from mock elicited and elicited cells at three time points: $6 \mathrm{~h}, 18 \mathrm{~h}$, and 5 day. Expression profile plots for the set of probes corresponding to each down-regulated library are shown: 5 day library probes. Expression is given as the fold-change in gene expression in the elicited culture over the un-elicited culture. Only results from probes showing a statistically significant change in expression $(P<0.05)$ are shown.

Additional file 8. Table S4. List of sequences obtained from the SSH libraries.

Additional file 9. Table S5. List of primer sets used for RT-PCR analysis.

\section{Competing interests}

The authors declare that they have no competing interests.

\section{Authors' contributions}

SKL analysed the data and drafted the manuscript; NB created the SSH library and performed macroarray analysis; RT-PCR analysis was carried out by BP; KV prepared the cell cultures and JN, SCR and ELW designed and mentored the study and gave final approval to the manuscript. All authors read and approved the final manuscript.

\section{Acknowledgements}

This study is financially supported by National Institutes of Health (R01 GM070852). The authors would like to thank Dr. Donna Gibson of the US Plant Soil and Nutrition Laboratory of the USDA for the Taxus cell cultures.

\section{Author details}

'Department of Biology, University of Massachusetts, Amherst, MA 01003 USA. ${ }^{2}$ Department of Biochemistry and Molecular Biology, University of Massachusetts, Amherst, MA 01003, USA. ${ }^{3}$ Department of Chemical Engineering, University of Massachusetts, Amherst, MA 01003, USA. ${ }^{4}$ Plant Biology Graduate Program, University of Massachusetts, Amherst, MA 01003 , USA.

Received: 12 August 2011 Accepted: 16 April 2012 Published: 24 April 2012

\section{References}

1. Farnsworth NR, Akerele O, Bingel AS, Soejarto DD, Guo Z: Medicinal plants in therapy. Bull World Health Organ 1985, 63(6):965-981.

2. Exposito O, Bonfill M, Moyano E, Onrubia M, Mirjalili MH, Cusido RM, Palazon J: Biotechnological production of taxol and related taxoids: current state and prospects. Anticancer Agents Med Chem 2009, 9(1):109-121.

3. Vongpaseuth K, Roberts SC: Advancements in the understanding of Paclitaxel metabolism in tissue culture. Curr Pharm Biotechnol 2007, 8(4):219-236.

4. Frense D: Taxanes: perspectives for biotechnological production. Appl Microbiol Biotechnol 2007, 73(6):1233-1240

5. Tabata $\mathrm{H}$ : Production of paclitaxel and the related taxanes by cell suspension cultures of Taxus species. Curr Drug Targets 2006, 7 (4):453-461.

6. Kolewe ME, Henson MA, Roberts SC: Characterization of aggregate size in Taxus suspension cell culture. Plant Cell Rep 2010, 29(5):485-494.

7. Vongpaseuth K, Nims E, St Amand M, Walker EL, Roberts SC: Development of a particle bombardment-mediated transient transformation system for Taxus spp. cells in culture. Biotechnol Prog 2007, 23(5):1180-1185.

8. Naill MC, Roberts SC: Culture of isolated single cells from Taxus suspensions for the propagation of superior cell populations. Biotechnol Lett 2005, 27(21):1725-1730

9. Roberts SC, Naill M, Gibson DM, Shuler ML: A simple method for enhancing paclitaxel release from Taxus canadensis cell suspension cultures utilizing cell wall digesting enzymes. Plant Cell Rep 2003, 21 (12):1217-1220.

10. Pestchanker $\amalg$, Roberts SC, Shuler ML: Kinetics of taxol production and nutrient use in suspension cultures of Taxus cuspidata in shake flasks and a Wilson-type bioreactor. Enzyme Microb Technol 1996, 19(4):256-260

11. Fett-Neto AG, Melanson SJ, Nicholson SA, Pennington Jل, Dicosmo F: Improved taxol yield by aromatic carboxylic acid and amino acid feeding to cell cultures of taxus cuspidata. Biotechnol Bioeng 1994, 44 (8):967-971.

12. Zhang MLX, Zhang J, Zhang S, Dong M, Huo C, Shi Q, Gu Y, Cong B: Taxanes from the leaves of Taxus cuspidata. Chem Nat Comp 2010, 46(1):53-58.

13. Wani MC, Taylor HL, Wall ME, Coggon P, McPhail AT: Plant antitumor agents. VI. The isolation and structure of taxol, a novel antileukemic and antitumor agent from Taxus brevifolia. J Am Chem Soc 1971, 93(9):2325-2327.

14. Hezari M, Ketchum RE, Gibson DM, Croteau R: Taxol production and taxadiene synthase activity in Taxus canadensis cell suspension cultures. Arch Biochem Biophys 1997, 337(2):185-190.

15. Walker K, Croteau R: Taxol biosynthetic genes. Phytochemistry 2001, 58(1):1-7.

16. Hefner J, Rubenstein SM, Ketchum RE, Gibson DM, Williams RM, Croteau R: Cytochrome P450-catalyzed hydroxylation of taxa-4(5),11(12)-diene to taxa4(20), 11(12)-dien-5alpha-ol: the first oxygenation step in taxol biosynthesis. Chem Biol 1996, 3(6):479-489.

17. Hefner J, Ketchum RE, Croteau R: Cloning and functional expression of a cDNA encoding geranylgeranyl diphosphate synthase from Taxus 
canadensis and assessment of the role of this prenyltransferase in cells induced for taxol production. Arch Biochem Biophys 1998, 360(1):62-74.

18. Walker K, Croteau R: Molecular cloning of a 10-deacetylbaccatin III-10-Oacetyl transferase cDNA from Taxus and functional expression in Escherichia coli. Proc Natl Acad Sci U S A 2000, 97(2):583-587.

19. Wildung MR, Croteau R: A cDNA clone for taxadiene synthase, the diterpene cyclase that catalyzes the committed step of taxol biosynthesis. J Biol Chem 1996, 271(16):9201-9204

20. Schoendorf A, Rithner CD, Williams RM, Croteau RB: Molecular cloning of a cytochrome P450 taxane 10 beta-hydroxylase cDNA from Taxus and functional expression in yeast. Proc Natl Acad Sci U S A 2001, 98(4):1501-1506.

21. Walker $K$, Long $R$, Croteau $R$ : The final acylation step in taxol biosynthesis: cloning of the taxoid C13-side-chain $\mathrm{N}$ benzoyltransferase from Taxus. Proc Natl Acad Sci U S A 2002, 99 (14):9166-9171.

22. Walker K, Fujisaki S, Long R, Croteau R: Molecular cloning and heterologous expression of the $\mathrm{C}-13$ phenylpropanoid side chain-CoA acyltransferase that functions in Taxol biosynthesis. Proc Natl Acad Sci U S A 2002, 99(20):12715-12720.

23. Jennewein S, Rithner CD, Williams RM, Croteau RB: Taxol biosynthesis: taxane 13 alpha-hydroxylase is a cytochrome P450-dependent monooxygenase. Proc Natl Acad Sci U S A 2001, 98(24):13595-13600.

24. Walker K, Croteau R: Taxol biosynthesis: molecular cloning of a benzoylCoA:taxane 2alpha-O-benzoyltransferase cDNA from taxus and functional expression in Escherichia coli. Proc Natl Acad Sci U S A 2000, 97(25):13591-13596.

25. Walker K, Schoendorf A, Croteau R: Molecular cloning of a taxa-4(20),11(12)dien-5alpha-ol-O-acetyl transferase cDNA from Taxus and functional expression in Escherichia coli. Arch Biochem Biophys 2000, 374(2):371-380.

26. Gundlach $\mathrm{H}$, Muller MJ, Kutchan TM, Zenk MH: Jasmonic acid is a signal transducer in elicitor-induced plant cell cultures. Proc Natl Acad Sci U S A 1992, 89(6):2389-2393

27. Nims E, Dubois CP, Roberts SC, Walker EL: Expression profiling of genes involved in paclitaxel biosynthesis for targeted metabolic engineering. Metab Eng 2006, 8(5):385-394.

28. Kai G, Zhao L, Zhang L, Li Z, Guo B, Zhao D, Sun X, Miao Z, Tang K: Characterization and expression profile analysis of a new CDNA encoding taxadiene synthase from Taxus media. J Biochem Mol Biol 2005, 38(6):668-675.

29. Naill MC, Roberts SC: Flow cytometric analysis of protein content in Taxus protoplasts and single cells as compared to aggregated suspension cultures. Plant Cell Rep 2005, 23(8):528-533.

30. Yukimune $Y$, Tabata $H$, Higashi $Y$, Hara $Y$ : Methyl jasmonate-induced overproduction of paclitaxel and baccatin III in Taxus cell suspension cultures. Nat Biotechnol 1996, 14(9):1129-1132.

31. Yukimune $Y$, Hara Y, Nomura E, Seto H, Yoshida S: The configuration of methyl jasmonate affects paclitaxel and baccatin III production in Taxus cells. Phytochemistry 2000, 54(1):13-17.

32. Wu Q, Sun C, Luo H, Li Y, Niu Y, Sun Y, Lu A, Chen S: Transcriptome analysis of Taxus cuspidata needles based on 454 pyrosequencing. Planta Med 2011, 77(4):394-400

33. da Hao C, Ge G, Xiao P, Zhang Y, Yang L: The First Insight into the Tissue Specific Taxus Transcriptome via Illumina Second Generation Sequencing. PLoS One 2011, 6(6):e21220.

34. Lee EK, Jin YW, Park JH, Yoo YM, Hong SM, Amir R, Yan Z, Kwon E, Elfick A Tomlinson S, et al: Cultured cambial meristematic cells as a source of plant natural products. Nat Biotechnol 2010, 28(11):1213-1217.

35. Naill MC, Roberts SC: Flow cytometric identification of Paclitaxelaccumulating subpopulations. Biotechnol Prog 2005, 21(3):978-983.

36. Huang $X$, Madan A: CAP3: A DNA sequence assembly program. Genome Res 1999, 9(9):868-877.

37. Ketchum REB, Gibson DM, Croteau RB, Shuler ML: The kinetics of taxoid accumulation in cell suspension cultures of Taxus following elicitation with methyl jasmonate. Biotechnol Bioeng 1999, 62(1):97-105.

38. Mirjalili N, Linden JC: Methyl jasmonate induced production of Taxol in suspension cultures of Taxus cuspidata: Ethylene interaction and induction models. Biotechnol Prog 1996, 12:110-118.

39. Yukimune $Y$, Tabata H, Higashi Y, Hara Y: Methyl Jasmonate-induced overproduction of paclitaxel and baccatin III in Taxus cell suspension cultures. Nat Biotechnol 1996, 14:1129-1132
40. Kim BJ, Gibson DM, Shuler ML: Relationship of viability and apoptosis to taxol production in Taxus sp. suspension cultures elicited with methyl jasmonate. Biotechnol Prog 2005, 21(3):700-707.

41. Weber H: Fatty acid-derived signals in plants. Trends Plant Sci 2002, 7:217-224

42. Hermsmeier D, Schittko U, Baldwin IT: Molecular interactions between the specialist herbivore Manduca sexta (Lepidoptera, Sphingidae) and its natural host Nicotiana attenuata. I. Large-scale changes in accumulation of growth- and defenese-related plant mRNAs. Plant Physiol 2001, 125:683-700.

43. Croteau R, Ketchum REB, Long RM, Kaspera R, Wildung MR: Taxol biosynthesis and molecular genetics. Phytochem Rev 2006, 5:75-97.

44. Ketchum REB, Croteau R: The Taxus metabolome and the elucidation of the Taxol biosynthetic pathway in cell suspension cultures. In Biotechnology in Agriculture and Forestry, Volume 57. Edited by Saito KD, Dixon RA, Willmitzer L. Berlin: Springer-Verlag; 2006:291-309.

45. Chau M, Croteau R: Molecular cloning and characterization of a cytochrome P450 taxoid 2alpha-hydroxylase involved in Taxol biosynthesis. Arch Biochem Biophys 2004, 427(1):48-57.

46. Walker K, Ketchum RE, Hezari M, Gatfield D, Goleniowski M, Barthol A Croteau R: Partial purification and characterization of acetyl coenzyme A: taxa-4(20),11(12)-dien-5alpha-ol O-acetyl transferase that catalyzes the first acylation step of taxol biosynthesis. Arch Biochem Biophys 1999, 364 (2):273-279

47. Spanu P, Reinhart D, Boller T: Analysis and cloning of the ethyleneforming enzyme from tomato by functional expression of its mRNA in Xenopus laevis oocytes. EMBO J 1991, 10:2007-2013.

48. Zhang $\mathrm{CH}, \mathrm{Wu}$ JY: Ethylene inhibitors enhance elicitor-induced paclitaxel production in suspension cultures of Taxus spp. cells. Enzyme Microb Technol 2003, 32:71-77.

49. van Loon LC, Rep M, Pieterse CM: Significance of inducible defenserelated proteins in infected plants. Annu Rev Phytopathol 2006, 44:135-162.

50. Lamb CJ, Dixon RA: Molecular mechanisms underlying induction of plant defence gene transcription. Biochem Soc Symp 1994, 60:241-248.

51. Ji SJ, Lu YC, Feng JX, Wei G, Li J, Shi YH, Fu Q, Liu D, Luo JC, Zhu YX Isolation and analyses of genes preferentially expressed during early cotton fiber development by subtractive PCR and CDNA array. Nucleic Acids Res 2003, 31(10):2534-2543.

52. Zheng J, Zhao J, Tao Y, Wang J, Liu Y, Fu J, Jin Y, Gao P, Zhang J, Bai Y, et al: Isolation and analysis of water stress induced genes in maize seedlings by subtractive PCR and cDNA macroarray. Plant Mol Biol 2004, 55(6):807-823.

53. Rebrikov DV, Britanova OV, Gurskaya NG, Lukyanov KA, Tarabykin VS, Lukyanov SA: Mirror orientation selection (MOS): a method for eliminating false positive clones from libraries generated by suppression subtractive hybridization. Nucleic Acids Res 2000, 28(20):E90.

54. Roberts SC: Production and engineering of terpenoids in plant cell culture. Nat Chem Biol 2007, 3(7):387-395.

55. Chirgwin JM, Przybyla AE, MacDonald RJ, Rutter WJ: Isolation of biologically active ribonucleic acid from sources enriched in ribonuclease. Biochemistry 1979, 18(24):5294-5299.

56. Dilger M, Felsenstein FG, Schwarz G: Identification and quantitative expression analysis of genes that are differentially expressed during conidial germination in Pyrenophora teres. Mol Genet Genomics 2003, 270 (2):147-155

57. Conesa A, Gotz S, Garcia-Gomez JM, Terol J, Talon M, Robles M: Blast2GO: a universal tool for annotation, visualization and analysis in functional genomics research. Bioinformatics 2005, 21(18):3674-3676.

58. Cleveland W: Robust locally weighted regression and smoothing scatter plots. J Am Stat Assoc 1979, 74(368):829-836.

doi:10.1186/1471-2164-13-148

Cite this article as: Lenka et al:: Identification and expression analysis of methyl jasmonate responsive ESTs in paclitaxel producing Taxus cuspidata suspension culture cells. BMC Genomics 2012 13:148. 